FORMATION Formation emploi

Revue française de sciences sociales

117 | janvier-mars 2012

Enseignement supérieur : les défis de la professionnalisation

\title{
L'insertion professionnelle après des études supérieures : des diplômés plus égaux que d'autres...
}

Employability after higher education: some graduates are more equal than others...

Berufliche Eingliederung nach dem Hochschulstudium: manche Abschliusse sind gleicher als andere...

La inserción profesional después de los estudios superiores: algunos graduados más iguales que otros...

\section{Julien Calmand et Dominique Epiphane}

\section{(2) OpenEdition Journals}

Édition électronique

URL : http://journals.openedition.org/formationemploi/3509

DOI : 10.4000/formationemploi.3509

ISSN : 2107-0946

Éditeur

La Documentation française

Édition imprimée

Date de publication : 23 avril 2012

Pagination : 11-28

ISSN : 0759-6340

Référence électronique

Julien Calmand et Dominique Epiphane, «L'insertion professionnelle après des études supérieures: des diplômés plus égaux que d'autres... », Formation emploi [En ligne], 117 | janvier-mars 2012, mis en ligne le 06 juin 2012, consulté le 30 octobre 2020. URL : http://journals.openedition.org/ formationemploi/3509; DOI : https://doi.org/10.4000/formationemploi.3509 


\title{
L'insertion professionnelle après des études supérieures : des diplômés plus égaux que d'autres...
}

\author{
Julien CALMAND \\ Économiste et ingénieur d'études au Céreq
}

Dominique EPIPHANE

Sociologue et ingénieure de recherche au Céreq

Résumé

- L'insertion professionnelle après des études supérieures : des diplômés plus égaux que d'autres...

Particulièrement vif dans les années soixante-dix, le débat sur la dimension méritocratique de l'école ressurgit aujourd'hui. La massification de l'enseignement supérieur ne s'est en effet pas accompagnée d'une réduction notable des inégalités. Dès lors, la question de sa démocratisation doit être appréciée à l'aune de la probabilité d'accéder aux filières les plus prestigieuses et ou les plus rentables sur le marché du travail. Dans un paysage où l'offre de formation s'est fortement enrichie, comment s'opèrent les différentes segmentations au sein de l'enseignement supérieur? Certains types de diplômes (comme les licences professionnelles) neutralisent plus que d'autres les effets de l'origine sociale sur les débuts de carrières de leurs détenteurs. Pour autant, les clivages en termes d'origine sociale n'ont pas disparu. Nous nous appuierons ici sur les données de l'enquête 2007 du Céreq, qui porte sur la Génération 2004.

Mots clés : jeune $\cdot$ diplôme $\bullet$ enseignement supérieur $\bullet$ insertion professionnelle $\bullet$ segmentation du marché du travail

Abstract

Employability after higher education: some graduates are more equal than others...

Debate on the meritocratic aspect of education, which was particularly fierce in the 1970s, is attracting renewed interest. Opening higher education up to the masses has not, in fact, been synonymous with any significant reduction in inequality. As a result, the question of its democratisation should be examined on the basis of the probability of access to the most prestigious courses and/or the courses that bring the highest returns on the labour market. At a time when education and training opportunities have vastly improved, 
how do the different segmentations within the higher education system operate? Certain qualifications (such as vocational degrees) offset the effects of social background on the early career path of the graduate more effectively than others. Even so, inequalities in terms of social background have not been eliminated. For this paper, we used data from the Céreq's 2007 Survey which covers the Generation of 2004.

Key words: transition from school to work $\bullet$ higher education $\bullet$ diploma $\bullet$ inequalities $\bullet$ social origin $\bullet$ school-to-work transition survey

Journal of Economic Literature: J 16, J 24

Traduction : Provence Traduction

Particulièrement vif dans les années soixante-dix, le débat sur la dimension méritocratique de l'école ressurgit aujourd'hui. En 1970, Pierre Bourdieu et Jean-Claude Passeron posaient la question en ces termes : l'école permet-elle à tous les enfants, quel que soit leur milieu social d'origine, d'obtenir une formation et une position sociale conformes à leur "mérite " ou n'est-elle qu'un instrument de reproduction et de légitimation des hiérarchies sociales? (Bourdieu et Passeron, 1970). Depuis, la terminologie a changé. Alors que les analyses en termes de "reproduction" (Bourdieu et Passeron, 1964, 1970) et d'" inégalité des chances" (Boudon, 1973) sont très présentes dans les années soixante-dix, ces appellations disparaissent progressivement au profit d'analyses en termes de "démocratisation" (Merle, 2002). Et même si la question de la " démocratisation" de l'enseignement supérieur en France est aujourd'hui souvent focalisée, dans le débat public, sur celle de la "démocratisation des Grandes Ecoles », elle renvoie néanmoins «à la perception, mal mesurée mais confuse en haut lieu, d'une aggravation de la reproduction sociale des classes supérieures» (Beaud et Convert, 2010).

La massification de l'enseignement supérieur est un phénomène engagé depuis plusieurs décennies. La forte hausse des effectifs au cours du siècle dernier a bouleversé le paysage. Après une évolution soutenue du nombre d'étudiants au cours de la première moitié du XXème siècle, cette croissance s'accélère dans les années soixante et va alors prendre une ampleur considérable puisque les effectifs ont été multipliés par six. Depuis la réforme de 1985 sur le baccalauréat et la proclamation de l'objectif « des 80 \% d'une classe d'âge au bac ", les effectifs de l'enseignement supérieur n'ont cessé d'augmenter et ont pratiquement doublé ces vingt dernières années ( $c f$. tableau $\mathbf{1})$.

Pour autant, la question de sa « démocratisation » reste d'actualité, l'allongement de la scolarité ayant déplacé le curseur pour la mesurer. Si aujourd'hui, plus de $50 \%$ d'une classe d'âge accède à l'enseignement supérieur, c'est désormais à l'aune de la probabilité d'entrer dans ses filières les plus prestigieuses et/ou les plus rentables sur le marché du travail, et d'y obtenir effectivement un diplôme, qu'elle doit être appréciée. Pour Stéphane Beaud 


\section{Tableau 1}

Evolution des effectifs d'étudiants en France (en milliers)

\begin{tabular}{|l|c|c|c|c|c|c|}
\hline & $\mathbf{1 9 8 0 - 8 1}$ & $\mathbf{1 9 9 0 - 9 1}$ & $\mathbf{2 0 0 0 - 0 1}$ & $\mathbf{2 0 0 4 - 0 5}$ & $\mathbf{2 0 0 9 - 2 0 1 0}$ & $\begin{array}{c}\text { Taux } \\
\text { d'évolution } \\
(\mathbf{1 9 8 0}-\mathbf{2 0 1 0 )}\end{array}$ \\
\hline CPGE & 42,9 & 68,4 & 73,8 & 76,5 & 85,5 & $99 \%$ \\
\hline STS & 67,9 & 199,3 & 238,9 & 230,3 & 240,3 & $254 \%$ \\
\hline IUT & 53,7 & 74,3 & 119,2 & 112,4 & 118,1 & $120 \%$ \\
\hline Universités & 790,1 & 1075,1 & 1254,3 & 1286,4 & 1306,1 & $65 \%$ \\
\hline Ecoles d'ingénieurs & 40 & 57,7 & 96,5 & 107,2 & 118,3 & $196 \%$ \\
\hline Ecoles de commerce & 15,8 & 46,1 & 63,4 & 83,2 & 116,3 & $636 \%$ \\
\hline Ecoles santé, social & 91,7 & 74,4 & 93,4 & 124,2 & 137,2 & $50 \%$ \\
\hline Autres & 76 & 128,5 & 232,4 & 236,7 & 205,3 & $170 \%$ \\
\hline Ensemble & 1184,7 & 1717,1 & 2160,3 & 2269,7 & 2316,1 & $96 \%$ \\
\hline
\end{tabular}

Source : RERS, MEN, 2009-2010.

Lecture : Pour l'année 1980-81, les effectifs des CPGE s'élevaient à 42900 inscrits.

Sigles : CPGE : Classes préparatoires aux grandes Écoles; STS : Sections de techniciens supérieurs ; IUT : Instituts universitaires de technologie.

(2003), la massification de l'enseignement supérieur a permis aux jeunes des milieux populaires d'accéder à l'université, mais le plus souvent dans les filières générales avec de moindres possibilités d'insertion sur le marché du travail. Selon Dominique Goux et Eric Maurin, l'expansion scolaire ne s'est pas accompagnée d'une réduction notable des inégalités et le niveau de diplôme des enfants est corrélé à celui du père comme il ne l'a jamais été (Goux et Maurin 1997). De même, pour Thomas Piketty, "le paradis perdu de l'ascenseur social tournant à plein régime" n'existe pas plus aujourd'hui qu'hier. La démocratisation de l'enseignement supérieur est un leurre car les "inégalités de réussite se sont simplement translatées vers le haut", elles sont devenues "moins lisibles " car "plus subtiles" (Piketty, 2006). De son côté, Pierre Merle constate que la distinction proposée par Antoine Prost (1986), dès les années quatre-vingt, entre démocratisation quantitative (le fait qu'une proportion croissante de membres d'une même classe d'âge accède à un niveau donné) et démocratisation qualitative (la réduction des inégalités sociales dans l'accès à ce niveau) n'est plus suffisamment heuristique pour décrire ce qui ce passe aujourd'hui. Selon lui, les travaux sur la démocratisation ne doivent plus se "focaliser sur l'allongement de la scolarisation et ses conséquences sur la place de chaque catégorie sociale, sans prendre en compte la différenciation de l'offre de formation" (Merle, 2000). Il est en effet devenu indispensable de prendre en compte la diversification des filières d'un niveau donné car toutes ne se valent pas sur le marché du travail. Ainsi par exemple, au niveau du baccalauréat, il qualifie la démocratisation de "ségrégative " car la hausse des taux de scolarisation à ce niveau, entre 1985 et 1995, s'est accompagnée d'une accentuation des écarts de recrutement social dans les différentes filières. De même, la « démocratisation » des études supérieures s'est opérée au prix d'un maintien, voire d'un renforcement, de la différenciation des filières. Selon Marie Duru-Bellat et Annick Kieffer, l'ouverture quantitative très nette de l'enseignement 
supérieur s'est accompagnée d'une différenciation sociale marquée des étudiants selon les filières et les disciplines et n'a pas contribué à réduire les inégalités sociales (Duru-Bellat et Kieffer, 2008).

Dans un paysage d'offre de formation qui s'enrichit, la présente étude se propose d'analyser la manière dont s'opèrent désormais les différentes segmentations de l'enseignement supérieur. Par exemple, dans un contexte où sa " professionnalisation " est à l'ordre $\mathrm{du}$ jour ${ }^{1}$ et où des filières universitaires telles que les licences et masters professionnels semblent relativement prisées et efficientes sur le marché du travail, il est intéressant de considérer les publics concernés. Nous tenterons de comprendre si ces évolutions récentes profitent à tous les publics ou si les anciens clivages en termes d'origine sociale sont toujours à l'œuvre. Nous verrons, dans un premier temps, comment se répartissent les jeunes dans l'enseignement supérieur selon leur origine sociale. Dans un deuxième temps, après avoir rapidement examiné les conditions d'insertion professionnelle à l'issue des principales filières de l'enseignement supérieur, nous tenterons d'apprécier si l'origine sociale a, à formation équivalente, un effet propre sur les débuts de parcours professionnels des jeunes diplômés.

\section{Des filières professionnelles socialement plus ouvertes}

Le capital scolaire détenu par les jeunes à la fin de leurs études initiales reste fortement conditionné par leur origine sociale ${ }^{2}$. La proportion de jeunes issus d'une famille de cadres augmente régulièrement avec le niveau de diplôme, tandis que celle des jeunes issus de parents ouvriers diminue. Très tôt, la reproduction sociale est à l'œuvre ; le débat actuel sur une démocratisation nécessaire de l'enseignement supérieur, avec notamment la question de la diversification des publics des grandes Écoles, semble bien être la dernière rangée d'arbres qui cache la forêt...

Dès l'enseignement secondaire, l'orientation se caractérise par des disparités sociales très marquées. À résultats scolaires comparables, l'orientation des élèves à la fin de la classe de $3^{\text {ème }}$ varie fortement en fonction de la profession de leurs parents : parmi les élèves ayant

1. Au cours des dernières années, l'offre de formation au niveau licence s'est très fortement développée : en 2004, plus de 1600 licences professionnelles étaient proposées en France, contre seulement 176 en 2000.

2. La position scolaire d'un jeune est mesurée par le plus haut diplôme obtenu avant de se présenter sur le marché du travail en 2004 ; son origine sociale, par la catégorie socioprofessionnelle occupée par son père au moment de sa sortie du système scolaire en 2004 (ou la dernière si celui-ci était au chômage, à la retraite ou décédé à ce moment-là). Nous savons que la PCS (profession et catégorie sociale) du père et de la mère a une influence différente sur l'accès au niveau d'études selon le type de niveau, la discipline et le genre (Boumadi et Lemistre, 2006) mais nous ne disposons pas, dans l'échantillon mobilisé, de l'information sur la PCS de la mère. 
des résultats moyens, ceux dont les parents sont cadres demandent systématiquement une seconde générale ou technologique tandis que ceux dont les parents sont ouvriers n'expriment qu'à $60 \%$ ce vou (Léger, 2006). Plus tard, à l'issue de la classe de seconde, les enfants de cadres sont plus souvent orientés en $1^{\text {ère }}$ générale que les enfants d'ouvriers et ce, à résultats scolaires comparables (Caille, 2005).

De même, et toujours à niveau scolaire équivalent, les élèves de terminale ne s'orientent pas dans les mêmes filières de l'enseignement supérieur : «Les élèves d'origine sociale favorisée visent en effet un niveau d'études plus élevé que les autres. À l'inverse, les élèves de milieu social défavorisé sont plus nombreux à envisager une sortie au niveau bac $+2 "$ (Nakhili, 2010).

Une fois le seuil de l'enseignement secondaire dépassé, la répartition au sein de l'appareil éducatif reste très contrastée selon l'origine sociale des étudiants. Celle-ci reste toujours fortement prédictive du niveau atteint dans l'échelle des diplômes : la proportion de jeunes dont le père est cadre est d'un cinquième pour l'ensemble de la génération qui est sortie du système scolaire en 2004 ; cette part est d'environ un quart chez les diplômés de bac +2 et dépasse la moitié chez les diplômés des écoles de commerce, d'ingénieurs, d'architecture et chez les docteurs. Sur l'ensemble de la génération de sortants en 2004, un enfant de cadre a environ quatre fois plus de chances de sortir de l'enseignement supérieur avec un master 2 (ou plus) qu'un enfant dont le père n'est pas cadre, et presque huit fois plus de chances qu'un enfant d'ouvrier.

Au sein de l'enseignement supérieur, c'est principalement à l'université que le recrutement social s'est élargi. Alors qu'en 1960, un fils de cadre supérieur avait vingt-huit fois plus de chances d'accéder à l'université qu'un fils d'ouvrier, il n'en a, en 1990, que sept fois plus (Euriat et Thélot 1995). Les "inégalités d'accès à l'université persistent donc, mais tendent à s'amenuiser, et l'on peut affirmer que jamais l'université n'a été autant démocratique qu'aujourd'hui, du point de vue de son recrutement social " (Felouzis, 2000). Relativement aux grandes Écoles, l'université accueille donc, au niveau licence comme au niveau master, davantage de jeunes d'origine modeste. Toutefois, certaines de ces filières universitaires semblent plus ouvertes socialement que d'autres. Ainsi, à ces niveaux, l'origine sociale des étudiants des filières professionnelles est, relativement, moins élevée que celle des étudiants des filières générales et recherche : on observe en effet des écarts significatifs entre ces deux types de filières dans leur propension à accueillir des jeunes dont le père n'est pas cadre ( +5 points au niveau licence et +13 points au niveau master), comme si les filières universitaires professionnalisées permettaient un accès plus large aux étudiants d'horizons sociaux plus diversifiés ( $c f$. tableau 2).

Ainsi, les jeunes de père non-cadre et diplômés d'une licence ou d'un master professionnel ont pu passer à travers les mailles du filet de la sélection sociale grâce à leur profil scolaire beaucoup plus proche de celui des enfants de cadres des mêmes filières que de celui des autres jeunes de leur catégorie sociale d'origine. Ainsi, tandis que, globalement, les enfants d'origine non-cadre sont nettement moins nombreux que les enfants de cadre à arriver à 
Tableau 2

Origine sociale des jeunes sortants du système scolaire en 2004

\begin{tabular}{|c|c|c|c|c|}
\hline & & Père cadre & $\begin{array}{l}\text { Père non } \\
\text { cadre }\end{array}$ & $\begin{array}{l}\text { Indice de } \\
\text { représentation } \\
\text { (base }=1)^{*}\end{array}$ \\
\hline \multirow{5}{*}{ 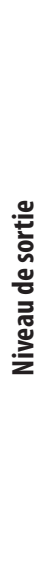 } & $\begin{array}{l}\text { Sorties de collège } \\
\text { Première année de CAP ou BEP }\end{array}$ & $\begin{array}{l}6 \\
7\end{array}$ & $\begin{array}{l}94 \\
93\end{array}$ & $\begin{array}{l}0,3 \\
0,4\end{array}$ \\
\hline & $\begin{array}{l}\text { CAP } \\
\text { BEP } \\
\text { Seconde, 1ère } \\
\text { 1ères professionnelles }\end{array}$ & $\begin{array}{c}7 \\
7 \\
16 \\
7\end{array}$ & $\begin{array}{l}93 \\
93 \\
84 \\
93\end{array}$ & $\begin{array}{l}0,4 \\
0,4 \\
0,8 \\
0,4\end{array}$ \\
\hline & $\begin{array}{l}\text { Bac général } \\
\text { Bac technologique, BT } \\
\text { Brevet professionnel } \\
\text { Bac professionnel }\end{array}$ & $\begin{array}{c}26 \\
15 \\
7 \\
12\end{array}$ & $\begin{array}{l}74 \\
85 \\
93 \\
88\end{array}$ & $\begin{array}{l}1,3 \\
0,8 \\
0,4 \\
0,6\end{array}$ \\
\hline & Ensemble Sortants du secondaire & 10 & 90 & 0,5 \\
\hline & $\begin{array}{l}\text { Après abandon ou échec BTS ou DUT } \\
\text { Après abandon ou échec DEUG }\end{array}$ & $\begin{array}{l}17 \\
24\end{array}$ & $\begin{array}{l}83 \\
76\end{array}$ & $\begin{array}{l}0,9 \\
1,2\end{array}$ \\
\hline \multirow{6}{*}{ 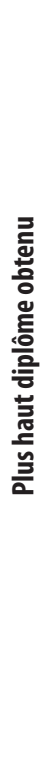 } & $\begin{array}{l}\text { BTS } \\
\text { DUT } \\
\text { DEUG-DEUST } \\
\text { Ecole d'Art Bac+2 } \\
\text { Bac+2 Santé-social }\end{array}$ & $\begin{array}{l}18 \\
25 \\
32 \\
37 \\
25\end{array}$ & $\begin{array}{l}82 \\
75 \\
68 \\
63 \\
75\end{array}$ & $\begin{array}{l}0,9 \\
1,3 \\
1,6 \\
1,9 \\
1,3\end{array}$ \\
\hline & $\begin{array}{l}\text { Licences générales } \\
\text { Licences professionnelles }\end{array}$ & $\begin{array}{l}31 \\
26\end{array}$ & $\begin{array}{l}69 \\
74\end{array}$ & $\begin{array}{l}1,6 \\
1,3\end{array}$ \\
\hline & $\begin{array}{l}\text { M1 universitaires } \\
\text { Ecole de commerce \& autres Bac+ } 4 \\
\text { MST - MSG y compris Maîtrise IUP } \\
\text { Ecole d'Art Bac+ } 4\end{array}$ & $\begin{array}{l}33 \\
37 \\
31 \\
46\end{array}$ & $\begin{array}{l}67 \\
63 \\
69 \\
54\end{array}$ & $\begin{array}{l}1,7 \\
1,9 \\
1,6 \\
2,3\end{array}$ \\
\hline & $\begin{array}{l}\text { Master Recherche et DEA } \\
\text { Master Pro et DESS } \\
\text { Ecoles de commerce Bac }+5 \\
\text { Ecoles d'Ingénieurs } \\
\text { Ecole d'architecture \& Autre Bac }+5\end{array}$ & $\begin{array}{l}49 \\
36 \\
55 \\
50 \\
52\end{array}$ & $\begin{array}{l}51 \\
64 \\
45 \\
50 \\
48\end{array}$ & $\begin{array}{l}2,5 \\
1,8 \\
2,8 \\
2,5 \\
2,6\end{array}$ \\
\hline & Doctorat & 52 & 48 & 2,6 \\
\hline & Ensemble Sortants du supérieur & 29 & 71 & 1,5 \\
\hline & Ensemble des jeunes de la cohorte & 20 & 80 & 1,0 \\
\hline
\end{tabular}

Source : Céreq - Génération 2004 interrrogée en 2007.

* Indice de représentation : lorsque l'indice est supérieur à 1, les enfants de cadre sont sur-représentés, Iorsqu'il est inférieur à 1, ils sont sous-représentés. 
Tableau 3

Profil scolaire des jeunes sortants de l'enseignement supérieur en 2004 (en \%)

\begin{tabular}{|c|c|c|c|c|c|c|c|c|}
\hline \multirow[b]{2}{*}{ Plus haut diplôme: } & \multicolumn{2}{|c|}{$\begin{array}{c}\text { En avance } \\
\text { ou " à l'heure " } \\
\text { en 6ème }\end{array}$} & \multicolumn{2}{|c|}{ Ont un bac général } & \multicolumn{2}{|c|}{ Ont un bac $S^{*}$} & \multicolumn{2}{|c|}{$\begin{array}{l}\text { Ont une mention } \\
\text { bien ou très bien* }\end{array}$} \\
\hline & $\begin{array}{l}\text { père } \\
\text { cadre }\end{array}$ & $\begin{array}{l}\text { père non } \\
\text { cadre }\end{array}$ & $\begin{array}{l}\text { père } \\
\text { cadre }\end{array}$ & $\begin{array}{l}\text { père non } \\
\text { cadre }\end{array}$ & $\begin{array}{l}\text { père } \\
\text { cadre }\end{array}$ & $\begin{array}{l}\text { père non } \\
\text { cadre }\end{array}$ & $\begin{array}{l}\text { père } \\
\text { cadre }\end{array}$ & $\begin{array}{l}\text { père non } \\
\text { cadre }\end{array}$ \\
\hline Licences générales & 97 & 96 & 91 & 87 & 45 & 31 & 9 & 8 \\
\hline Licences professionnelles & 96 & 96 & 59 & 52 & 55 & 60 & 3 & 4 \\
\hline Master Recherche et DEA & 98 & 95 & 97 & 95 & 49 & 40 & 7 & 4 \\
\hline Master Pro et DESS & 97 & 96 & 94 & 90 & 50 & 52 & 8 & 4 \\
\hline Ecoles de commerce $\mathrm{Bac}+5$ & 94 & 98 & 91 & 85 & 59 & 40 & 8 & 12 \\
\hline Ecoles d'Ingénieurs & 98 & 97 & 94 & 90 & 97 & 98 & 28 & 19 \\
\hline Ecole d'architecture \& Autre Bac +5 & 92 & 88 & 92 & 81 & 63 & 62 & 24 & 16 \\
\hline Doctorat & 98 & 96 & 99 & 96 & 84 & 79 & 19 & 8 \\
\hline Ensemble des sortants du supérieur & 96 & 93 & 77 & 56 & 56 & 45 & 13 & 9 \\
\hline Ensemble des jeunes de la cohorte & 93 & 82 & 58 & 26 & 56 & 45 & 13 & 9 \\
\hline
\end{tabular}

$\left.{ }^{*}\right)$ : parmi les bacheliers généraux.

Source : Céreq - Génération 2004 interrrogée en 2007.

Lecture : 91 \% des jeunes entrés sur le marché du travail en 2004 avec, comme plus haut diplôme, une Licence générale et dont le père est cadre, étaient titulaires d'un bac général.

Sigles : DEA : diplôme d'études approfondies; DESS : diplôme d'études supérieures spécialisées.

l'heure ou en avance en classe de $6^{\text {ème }}$ lorsqu'ils sont diplômés d'une licence ou d'un master professionnel, ils ont un profil tout à fait comparable à ces derniers ( $c f$. tableau 3). Leur propension à posséder un baccalauréat général est très proche ; de surcroît, quand ils sont bacheliers généraux, c’est plus souvent dans la série scientifique : $60 \%$ des licenciés professionnels dont le père n'est pas cadre sont titulaires d'un baccalauréat scientifique contre $55 \%$ des enfants de cadres. La même tendance est à l'œuvre chez les diplômés de master professionnel, avec un écart légèrement plus faible.

En revanche, leur parcours dans l'enseignement supérieur pour accéder au niveau L (licence) ou M (master) diffère quelque peu. En ce qui concerne les voies d'accès à ces filières professionnelles de l'université, nous pouvons observer que les enfants de noncadre y accèdent un peu moins souvent que ceux de cadre via le $1^{\text {er }}$ cycle universitaire et un peu plus souvent via un BTS ( $c f$. tableau 4).

La mise en place des licences professionnelles a bel et bien permis aux étudiants de bac +2 professionnel de poursuivre leurs études. En 2004, 55 \% des enfants dont les parents ne sont pas cadres, sortant de licence professionnelle, étaient également détenteurs d'un BTS ; c'est le cas pour $44 \%$ des enfants de cadres qui sont, quant à eux, un peu plus fréquemment issus d'un IUT. 
Tableau 4

Diplômes Bac+2 des diplômés de niveaux Licence, Master et Doctorat

\begin{tabular}{|l|c|c|c|c|c|c|}
\hline & \multicolumn{2}{|c|}{$\begin{array}{c}\text { Possède également } \\
\text { un BTS }\end{array}$} & \multicolumn{2}{c|}{$\begin{array}{c}\text { Possède également } \\
\text { un DUT }\end{array}$} & $\begin{array}{c}\text { Possède également } \\
\text { un Deug (ou Deust) }\end{array}$ \\
\cline { 2 - 7 } & père cadre & $\begin{array}{c}\text { père non } \\
\text { cadre }\end{array}$ & père cadre & $\begin{array}{c}\text { père non } \\
\text { cadre }\end{array}$ & père cadre & $\begin{array}{c}\text { père non } \\
\text { cadre }\end{array}$ \\
\hline Plus haut diplôme: & 5 & 7 & 3 & 5 & 74 & 73 \\
\hline Licences générales & 44 & 55 & 39 & 35 & 10 & 6 \\
Licences professionnelles & 2 & 3 & 8 & 7 & 66 & 70 \\
\hline Master Recherche et DEA & 3 & 8 & 15 & 22 & 71 & 62 \\
Master Pro et DESS & 5 & 16 & 9 & 23 & 20 & 18 \\
Ecoles de commerce Bac+5 & 4 & 10 & 15 & 24 & 25 & 18 \\
Ecoles d'Ingénieurs & 5 & 21 & 3 & 5 & 17 & 8 \\
\hline Ecole d'architecture \& Autre Bac+5 & 1 & 3 & 3 & 5 & 43 & 52 \\
\hline Doctorat & $\mathbf{8}$ & $\mathbf{1 4}$ & $\mathbf{9}$ & $\mathbf{1 1}$ & $\mathbf{3 9}$ & $\mathbf{3 5}$ \\
\hline Ensemble des sortants du supérieur & & & & & & 73 \\
\hline
\end{tabular}

Source : Céreq - Génération 2004 interrrogée en 2007.

Lecture : 5 \% des jeunes entrés sur le marché du travail en 2004 avec, comme plus haut diplôme, une licence générale et dont le père était cadre, possèdaient également un BTS.

\section{Licences et masters professionnels: une entrée dans la vie active relativement aisée}

L'analyse de l'insertion des diplômés des filières professionnelles au niveau licence comme au niveau master montre une réelle efficacité de ces diplômes sur le marché du travail relativement à leurs homologues des filières générales (Calmand et alii, 2009).

En 2007, le taux de chômage des licenciés professionnels est inférieur de 4 points à celui des licenciés généraux qui n'ont pas poursuivi leurs études à l'IUFM (Institut de formation des maîtres). À cette même date, la grande majorité bénéficie d'un contrat à durée indéterminée. La part de ceux et celles qui travaillent à temps partiel est très faible, y compris à l'issue des filières tertiaires. À ce niveau de sortie, les diplômés des filières professionnelles sont également mieux rémunérés que leurs homologues des filières générales : leurs salaires médians sont supérieurs de 200 euros. À la différence des filières générales, les filières professionnelles semblent donc constituer un atout pour les jeunes désirant se présenter directement sur le marché du travail sans poursuivre d'études supérieures au-delà de la licence. Si cet avantage sur le marché du travail est net au niveau licence, il l'est tout autant au niveau master, les diplômés de certaines filières telles que l'informatique ${ }^{3}$ connaissant

3. Très prisés sur le marché du travail, ces derniers connaissent très peu de chômage et occupent, pour $85 \%$ d'entre eux, des emplois de cadres, ce qui les place en tête des jeunes universitaires les mieux rémunérés avec un salaire médian net mensuel de 2000 euros. 
Tableau 5

Situation professionnelle en 2007 des jeunes diplômés de l'enseignement supérieur

\begin{tabular}{|c|c|c|c|c|c|c|}
\hline Plus haut diplôme: & $\begin{array}{l}\text { Taux de } \\
\text { chômage }\end{array}$ & $\begin{array}{c}\text { Part des } \\
\text { EDI* }\end{array}$ & $\begin{array}{l}\text { Part de } \\
\text { temps } \\
\text { partiel }\end{array}$ & $\begin{array}{l}\text { Taux de } \\
\text { cadres }\end{array}$ & $\begin{array}{c}\text { Taux de } \\
\text { PI** }\end{array}$ & $\begin{array}{l}\text { Salaire } \\
\text { médian }\end{array}$ \\
\hline BTS & $8 \%$ & $71 \%$ & $8 \%$ & $5 \%$ & $49 \%$ & $1310 €$ \\
\hline de spécialité industrielle & $6 \%$ & $73 \%$ & $4 \%$ & $5 \%$ & $54 \%$ & $1400 €$ \\
\hline de spécialité tertiaire & $10 \%$ & $70 \%$ & $11 \%$ & $5 \%$ & $45 \%$ & $1260 €$ \\
\hline DUT & $8 \%$ & $76 \%$ & $6 \%$ & $11 \%$ & $56 \%$ & $1410 €$ \\
\hline de spécialité industrielle & $7 \%$ & $72 \%$ & $3 \%$ & $8 \%$ & $68 \%$ & $1500 €$ \\
\hline de spécialité tertiaire & $9 \%$ & $78 \%$ & $7 \%$ & $12 \%$ & $49 \%$ & $1370 €$ \\
\hline DEUG-DEUST & $12 \%$ & $63 \%$ & $20 \%$ & $20 \%$ & $43 \%$ & $1260 €$ \\
\hline en sciences dures & $16 \%$ & $71 \%$ & $15 \%$ & $20 \%$ & $54 \%$ & $1450 €$ \\
\hline en droit, économie, gestion & $12 \%$ & $62 \%$ & $20 \%$ & $23 \%$ & $37 \%$ & $1260 €$ \\
\hline en lettres et sciences humaines & $9 \%$ & $62 \%$ & $27 \%$ & $10 \%$ & $53 \%$ & $1200 €$ \\
\hline Licences générales (hors IUFM) & $10 \%$ & $62 \%$ & $23 \%$ & $12 \%$ & $61 \%$ & $1300 €$ \\
\hline en sciences dures & $7 \%$ & $69 \%$ & $16 \%$ & $15 \%$ & $67 \%$ & $1400 €$ \\
\hline en droit, économie, gestion & $10 \%$ & $72 \%$ & $15 \%$ & $17 \%$ & $52 \%$ & $1400 €$ \\
\hline en lettres et sciences humaines & $9 \%$ & $61 \%$ & $27 \%$ & $12 \%$ & $64 \%$ & $1260 €$ \\
\hline Licences professionnelles & $6 \%$ & $81 \%$ & $3 \%$ & $13 \%$ & $65 \%$ & $1500 €$ \\
\hline de spécialité industrielle & $5 \%$ & $81 \%$ & $2 \%$ & $10 \%$ & $72 \%$ & $1540 €$ \\
\hline de spécialité tertiaire & $6 \%$ & $80 \%$ & $5 \%$ & $15 \%$ & $58 \%$ & $1470 €$ \\
\hline M1 universitaires & $9 \%$ & $70 \%$ & $13 \%$ & $32 \%$ & $49 \%$ & $1500 €$ \\
\hline sciences dures (y compris santé, SVT) & $5 \%$ & $71 \%$ & $9 \%$ & $52 \%$ & $39 \%$ & $1550 €$ \\
\hline langues, lettres, arts & $8 \%$ & $69 \%$ & $15 \%$ & $37 \%$ & $49 \%$ & $1500 €$ \\
\hline sciences humaines, psychologie et éducation & $7 \%$ & $62 \%$ & $18 \%$ & $27 \%$ & $54 \%$ & $1450 €$ \\
\hline économie, gestion, communication & $12 \%$ & $77 \%$ & $8 \%$ & $25 \%$ & $48 \%$ & $1500 €$ \\
\hline droit, sciences politiques & $11 \%$ & $74 \%$ & $11 \%$ & $23 \%$ & $52 \%$ & $1430 €$ \\
\hline Master Recherche et DEA & $10 \%$ & $74 \%$ & $12 \%$ & $56 \%$ & $33 \%$ & $1680 €$ \\
\hline en sciences dures & $12 \%$ & $70 \%$ & $8 \%$ & $57 \%$ & $37 \%$ & $1700 €$ \\
\hline en droit, économie, gestion & $7 \%$ & $86 \%$ & $5 \%$ & $64 \%$ & $29 \%$ & $1850 €$ \\
\hline en lettres et sciences humaines & $13 \%$ & $61 \%$ & $27 \%$ & $44 \%$ & $37 \%$ & $1450 €$ \\
\hline Master Pro et DESS & $7 \%$ & $79 \%$ & $6 \%$ & $61 \%$ & $31 \%$ & $1820 €$ \\
\hline en sciences dures & $8 \%$ & $79 \%$ & $4 \%$ & $69 \%$ & $26 \%$ & $1900 €$ \\
\hline en droit, économie, gestion & $5 \%$ & $84 \%$ & $3 \%$ & $60 \%$ & $32 \%$ & $1900 €$ \\
\hline en lettres et sciences humaines & $8 \%$ & $66 \%$ & $16 \%$ & $53 \%$ & $37 \%$ & $1500 €$ \\
\hline Ecoles de commerce Bac+5 & $6 \%$ & $93 \%$ & $3 \%$ & $65 \%$ & $27 \%$ & $2300 €$ \\
\hline Ecoles d'Ingénieurs & $4 \%$ & $92 \%$ & $1 \%$ & $87 \%$ & $10 \%$ & $2150 €$ \\
\hline Doctorat & $6 \%$ & $72 \%$ & $12 \%$ & $92 \%$ & $7 \%$ & $2170 €$ \\
\hline en médecine, pharmacie & $2 \%$ & $74 \%$ & $15 \%$ & $97 \%$ & $3 \%$ & $2520 €$ \\
\hline en sciences dures (hors santé) & $9 \%$ & $70 \%$ & $5 \%$ & $91 \%$ & $98 \%$ & $2050 €$ \\
\hline en droit, économie, gestion & $6 \%$ & $81 \%$ & $10 \%$ & $92 \%$ & $6 \%$ & $2100 €$ \\
\hline en lettres et sciences humaines & $10 \%$ & $68 \%$ & $21 \%$ & $79 \%$ & $17 \%$ & $1840 €$ \\
\hline Ensemble des sortants du supérieur & $8 \%$ & $74 \%$ & $10 \%$ & $25 \%$ & $47 \%$ & $1500 €$ \\
\hline
\end{tabular}

Source : Céreq - Génération 2004 interrrogée en 2007.

Lecture : En 2007, le taux de chômage des jeunes entrés sur le marché du travail en 2004 avec, comme plus haut diplôme, une licence générale, était de $10 \%$. $\left(^{*}\right)$ : emploi à durée indéterminée $\left(^{* *}\right)$ : professions intermédiaires.

Sigles : BTS: brevet de technicien supérieur;DUT: diplôme universitaire de technologie;DEUG: diplôme détudes universitaires générales; DEUST: diplôme d'études universitaires scientifiques et techniques ; DEA : diplôme détudes approfondies; DESS : diplôme détudes supérieures spécialisées ; IUFM : Institut universitaire de formation des maîtres. 
des modalités d'insertion tout à fait comparables à celles des sortants d'écoles d'ingénieurs. Toutes filières confondues, plus de $61 \%$ des jeunes sortants de master professionnel sont cadres au moment de l'enquête, leur taux de chômage à trois ans est inférieur de 3 points à celui des masters des filières générales, leur part d'EDI ${ }^{4}$ supérieure de 5 points et leurs salaires médians supérieurs de 140 euros ( $c f$. tableau 5).

Cet avantage des diplômés des filières professionnelles peut également se mesurer à l'aune du " déclassement" relatif des licenciés généraux (Affichard, 1981). En effet, les jeunes entrant sur le marché du travail avec leur seule licence générale en poche sont plus nombreux à occuper des emplois d'employés ou ouvriers que les licenciés professionnels. On observe ce même phénomène au niveau master. Par ailleurs, d'un point de vue plus subjectif, les licenciés généraux, en emploi en 2007, ont plus souvent le sentiment d'être employés en dessous de leur niveau de compétences que les jeunes diplômés de licence professionnelle. De la même façon, les diplômés de master des filières générales sont moins satisfaits de l'emploi qu'ils exercent que ceux des filières professionnelles.

Alors que les diplômes généraux ont été longtemps critiqués pour leur distance par rapport aux besoins du marché du travail (Godet, 1993), les diplômes professionnels sont supposés permettre de transformer les savoirs académiques en attitudes et en compétences plus proches des besoins des entreprises. Mais surtout, en intégrant une multitude d'expériences obligatoires de travail pendant les études - par l'intermédiaire des stages par exemple - conjugué à un lien plus fort avec le "monde de l'entreprise ", les filières professionnelles sont censées permettre aux jeunes dotés d'un faible capital social d'en acquérir davantage. En comparaison des jeunes issus des milieux plus favorisés, leurs relations familiales mobilisables au moment de l'insertion professionnelle, généralement concentrées sur les segments d'emplois peu qualifiés, sont peu efficientes ; dès lors, les diplômes professionnels permettraient de palier ce handicap social en apportant davantage de ressources et de liens avec le marché du travail. Dans la partie suivante, nous étudierons donc si les conditions d'insertion et les cibles professionnelles atteintes des diplômés de ces différentes filières sont proches, quelles que soient leurs caractéristiques individuelles et, notamment, leur origine sociale.

\section{L'origine sociale n'a pas d'effet significatif sur l'accès à l'emploi}

L'analyse de la situation professionnelle des jeunes diplômés, trois ans après leur sortie de l'enseignement supérieur, permet de mesurer, au-delà des différentes séquences d'emplois occupés, des diverses expériences professionnelles rencontrées pendant leurs premières

4. L'emploi à durée indéterminée est constitué par les emplois de fonctionnaires et les emplois sous CDI (contrat à durée indéterminée). 


\section{Encadré 1}

\section{Méthodologie}

Au printemps 2007, le Céreq a interrogé un échantillon national d'environ 34000 individus sortis de formation initiale entre octobre 2003 et octobre 2004 (et, pour les docteurs, jusqu'en décembre 2004) ; il a été complété par diverses extensions d'échantillon représentant environ 25000 individus supplémentaires. Ces individus sont issus de tous les niveaux de formation et font partie des 737000 jeunes qui, cette année là, ont quitté pour la première fois le système éducatif.

L'enquête 2007 sur la « Génération 2004 » fait suite aux enquêtes « Génération » précédentes (1992, 1998 et 2001). Elle poursuit le même objectif : analyser les trois premières années de vie active des jeunes sortants du système éducatif.

Elle privilégie toujours une approche longitudinale de l'insertion. Elle permet à chaque jeune de retracer son parcours professionnel, depuis sa sortie du système éducatif, en 2003/2004, jusqu'au printemps 2007, soit environ trois ans. Grâce à un calendrier mensuel, le jeune reconstitue son parcours. En fonction des situations décrites dans ce calendrier - emploi, chômage, inactivité, reprise d'études ou formation -, des modules de questions lui sont ensuite soumis automatiquement.

Pour cette étude, nous avons travaillé sur l'ensemble des diplômés de l'enseignement supérieur (à l'exception des diplômés des écoles d'art, de santé, du social, d'architecture) entrés sur le marché du travail en 2004. Ces jeunes français ou étrangers ont moins de 35 ans au moment de l'interrogation et ils n'avaient pas interrompu leurs études une année ou plus avant l'année scolaire 2003-2004. Ils n'ont pas repris leurs études pendant l'année qui a suivi leur entrée sur le marché du travail. Au final, notre échantillon se compose d'environ 25000 individus représentant les 240000 jeunes sortis en 2004 de l'enseignement supérieur avec un diplôme en poche (hors diplômés des écoles d'art, de santé, du social, d'architecture).

\section{Modèles Probit utilisés :}

Dans cet article, nous cherchons à vérifier si l'origine sociale a un effet sur l'insertion, c'est-à-dire sur l'accès à l'emploi mais aussi sur la nature de cet emploi. Nous allons donc tester ces hypothèses, grâce à des estimations dites « toutes choses égales par ailleurs » (c'est-à-dire à caractéristiques connues comparables). Nous travaillons sur la situation professionnelle des diplômés de l'enseignement supérieur trois années après leur sortie du système éducatif. Nous savons que l'origine sociale $a$ un effet sur l'accès à l'emploi de cadre au premier emploi (Boumadhi, Lemistre 2006), et nous souhaitons vérifier si l'origine sociale joue encore sur le fait d'être en emploi, d'être en emploi à durée indéterminée (emplois de fonctionnaires et emplois sous CDI) et d'accéder aux professions intermédiaires ou cadres, trois ans après la sortie de l'enseignement supérieur.

\section{Variables explicatives :}

Une première série de variables concerne le profil sociodémographique des individus interrogés, comme le sexe, I'origine sociale et le lieu de résidence au moment de l'enquête. Nous avons appréhendé l'origine sociale des jeunes à travers la catégorie socioprofessionnelle (PCS) de leur père (ou la dernière si celui-ci était au chômage, à la retraite ou décédé à ce moment-là). Nous savons que la PCS du père et de la mère a une influence différente sur l'accès au niveau d'études selon le type de niveau, la discipline et le genre (Boumadi et Lemistre, 2006). Par ailleurs, il existe d'autres variables robustes comme l'accès ou non à une bourse sur critère social pour financer ses études, le niveau d'études des parents... mais nous ne disposons pas, dans l'échantillon mobilisé, de ces informations. De plus, si la catégorie " cadre » est hétérogène, elle reste cependant une variable proxi acceptable de l'origine sociale et peut expliquer la probabilité d'être en emploi mais aussi renseigner sur le niveau d'étude atteint.

Une deuxième batterie de variables explicatives concerne le parcours scolaire des individus. Nous avons introduit deux variables explicatives : le type de bac et le fait d'être passé par une classe préparatoire directement après le bac. Enfin, une série de variables nous renseigne sur le plus haut diplôme des jeunes sortis en 2004. Nous avons également introduit, dans le modèle, la spécialité du diplôme, déclinée en trois catégories : lettres et sciences humaines, droit et économie gestion et sciences dures. 
années de vie active, les effets éventuels de l'origine sociale à cette étape de leur début de carrière. Il s'agit donc de déterminer dans un premier temps si, à filière équivalente, subsiste un effet de l'origine des jeunes sur leur positionnement sur le marché du travail ou, plus exactement, sur la probabilité d'occuper, trois ans après la sortie de formation, un emploi, voire un emploi à durée indéterminée.

L'analyse de ce positionnement, toutes choses égales par ailleurs (c'est-à-dire à caractéristiques connues comparables), rend compte d'une série de résultats classiquement observés depuis plusieurs générations de jeunes diplômés. La probabilité d'être effectivement en emploi ${ }^{5}$, trois ans après la sortie du système éducatif est, avant tout, fortement corrélée au niveau et au type de diplôme obtenu ( $c f$. tableau 6). Cette probabilité est maximale pour les diplômés des écoles d'ingénieurs et de commerce ainsi que, assez logiquement, pour les sortants d'IUFM. Au niveau bac + 2, les sortants de L2 (diplômés de DEUG - diplôme d'études universitaires générales) ont une probabilité moins importante d'être en emploi que ceux de BTS ou de DUT (respectivement brevet de technicien supérieur et diplôme universitaire de technologie), tout comme les diplômés d'une licence générale. Au-delà de l'effet " niveau ", on observe donc, toutes choses égales par ailleurs, un avantage notable pour les diplômés des filières professionnelles. Quel que soit le niveau de diplôme, la probabilité d'être en emploi en 2007 est plus forte pour les diplômés des filières professionnelles que pour ceux des filières générales : c'est ainsi le cas pour les diplômés de BTS ou de DUT par rapport à ceux de DEUG, pour ceux de licences ou de masters professionnels relativement à ceux de licences ou de masters disciplinaires. Par ailleurs, le sexe est fortement corrélé au fait d'être en emploi, trois ans après les premiers pas sur le marché du travail : être un homme augmente en effet de $11 \%$ cette probabilité. De même, quand on examine la nature des postes occupés, il s'avère que les hommes ont plus de chances d'occuper un emploi de cadre ou de profession intermédiaire que les femmes (avec un différentiel assez fort pour l'accès à la catégorie cadre). Ce résultat confirme d'autres études qui ont montré que les jeunes hommes accédaient, dès leurs premières années de vie active, plus fréquemment à cette catégorie que les jeunes femmes (Epiphane, 2002 ; Couppié et Epiphane, 2008 ; Guégnard et alii, 2008).

Quant à l'effet de l'origine sociale, mesurée par la catégorie sociale (CS) du père, il est nul sur cet indicateur : être enfant de cadre n'augmente pas la probabilité d'être en emploi trois années après la sortie de l'enseignement supérieur. De même, lorsque l'on prend en compte le statut de l'emploi occupé, l'origine sociale n'a, là aussi, aucun effet significatif sur la probabilité que cet emploi soit à durée indéterminée ${ }^{6}$.

5. Dans ce modèle, nous avons mesuré la probabilité d'être en emploi versus celle d'être au chômage, inactif ou d'être retourné en formation en 2007, soit trois ans après la sortie de formation.

6. Ici, le modèle est calculé sur la population active occupée au moment de l'enquête. L'emploi à durée indéterminée est constitué par les emplois de fonctionnaires et les emplois sous CDI. Afin d'assurer une comparabilité dans les modèles, nous avons gardé les mêmes variables explicatives que dans le modèle précédent. 
Tableau 6

Modèle Probit à variables dépendantes (emploi, statut)

\begin{tabular}{|c|c|c|c|c|}
\hline & \multicolumn{4}{|c|}{ En 2007, être: } \\
\hline & en emploi & en EDI $(*)$ & $\begin{array}{l}\text { P.I. }\left(^{* *}\right) \\
\text { ou cadre }\end{array}$ & cadre \\
\hline Constante & +++ & +++ & ns & -- \\
\hline Homme (Ref Femme) & +++ & +++ & +++ & +++ \\
\hline Région de Residence : Rural & - & - & ns & ns \\
\hline $\begin{array}{l}\text { Bac d'origine (Ref: Bac Général) } \\
\text { Bac Autre } \\
\text { Bac professionnel } \\
\text { Bac technologique }\end{array}$ & $\begin{array}{c}\text { ns } \\
+++ \\
-\end{array}$ & $\begin{array}{l}+++ \\
+++ \\
\text { ns }\end{array}$ & $\begin{array}{l}\text { ns } \\
-- \\
--\end{array}$ & $\begin{array}{l}\text { ns } \\
\text { ns } \\
-\end{array}$ \\
\hline Avoir fait une classe prépa après le Bac & ns & +++ & ++ & +++ \\
\hline $\begin{array}{l}\text { Cs du Père (Ref: Cadre) } \\
\text { Ouvrier } \\
\text { Technicien } \\
\text { Employé } \\
\text { Autre } \\
\text { Artisan } \\
\text { Agriculteur }\end{array}$ & $\begin{array}{l}\text { ns } \\
\text { ns } \\
\text { ns } \\
-- \\
\text { ns } \\
\text { ns }\end{array}$ & $\begin{array}{l}\text { ns } \\
\text { ns } \\
\text { ns } \\
\text { ns } \\
\text { ns } \\
\text { ns }\end{array}$ & $\begin{array}{c}--- \\
\text { ns } \\
--- \\
\text { ns } \\
-- \\
---\end{array}$ & $\begin{array}{l}--- \\
--- \\
--- \\
\text { ns } \\
--- \\
---\end{array}$ \\
\hline $\begin{array}{l}\text { Spécialités (Réf: Droit Eco-Gestion) } \\
\text { Sciences dures } \\
\text { Lettres et sciences humaines }\end{array}$ & ns & --- & $\begin{array}{l}+++ \\
+++\end{array}$ & - \\
\hline $\begin{array}{l}\text { Plus haut diplôme (Ref: BTS) } \\
\text { DUT } \\
\text { DEUG } \\
\text { Licence générale } \\
\text { Licence pro } \\
\text { M1 } \\
\text { IUFM } \\
\text { DESS } \\
\text { DEA } \\
\text { Ecoles de commerce } \\
\text { Ecoles d'ingénieurs } \\
\text { Doctorat }\end{array}$ & $\begin{array}{c}\text { ns } \\
--- \\
--- \\
+++ \\
--- \\
+++ \\
+++ \\
--- \\
++ \\
+++ \\
+\end{array}$ & $\begin{array}{c}\text { ns } \\
--- \\
--- \\
+++ \\
- \\
+++ \\
+++ \\
\text { ns } \\
+++ \\
+++ \\
---\end{array}$ & $\begin{array}{c}+++ \\
\mathrm{ns} \\
+++ \\
+++ \\
+++ \\
+++ \\
+++ \\
+++ \\
+++ \\
+++ \\
+++\end{array}$ & $\begin{array}{l}+++ \\
+++ \\
+++ \\
+++ \\
+++ \\
+++ \\
+++ \\
+++ \\
+++ \\
+++ \\
+++\end{array}$ \\
\hline
\end{tabular}

Source : Céreq - Génération 2004 interrrogée en 2007.

Lecture : Les coefficients du modèles probit n'étant pas interprétables, la lecture des résultats dans les tableaux suivants est simplifiée à l'aide des signes «+» et «-» qui indiquent l'intensité du paramètre considéré pour tous les coefficients statistiquement significatifs au seuil de $10 \%$.

«ns» indique que le coefficient n'était pas significatif à ce seuil. Être un homme augmente la probabilité dêtre en emploi 3 années après la sortie du système éducatif, ce résultat est significatif au seuil de $10 \%$.

$\left({ }^{*}\right)$ : emploi à durée indéterminée. $\left.{ }^{* *}\right)$ : professions intermédiaires.

Sigles : Cs: catégorie sociale ; BTS : brevet de technicien supérieur ; DUT : diplôme universitaire de technologie ; DEUG : diplôme d'études universitaires générales ; M1 : master 1;DEA : diplôme d'études approfondies; DESS : diplôme d'études supérieures spécialisées ; IUFM : Institut universitaire de formation des maîtres. 
Sur cet indicateur, les effets du diplôme et de la spécialité sont également très importants ; les diplômes dits professionnels garantissant nettement plus souvent un accès à l'emploi à durée indéterminée relativement aux diplômes dits généraux. Les diplômés de licence professionnelle ont plus de chances d'accéder à ce type d'emploi que les diplômés de BTS, ce résultat étant très significatif. En revanche, les diplômés de DEUG, de licence générale et de maîtrise ont, quant à eux, moins de chances d'accéder à ce statut d'emploi. À noter que pour les diplômés de doctorat, cette probabilité est également faible en raison de leur forte propension à être, trois années après leur doctorat, encore engagés dans des expériences post-doctorales (Calmand et Giret, 2010).

\section{L'origine sociale a un effet significatif sur l'accès aux professions intermédiaires et cadres}

Être enfant de cadre n'augmente ni la probabilité d'être en emploi, ni celle d'occuper un emploi à durée indéterminée trois ans après la sortie de l'enseignement supérieur ; mais qu'en est-il de l'accès aux catégories de cadre ou de profession intermédiaire ? Ainsi, pour chacun des diplômes du supérieur, la probabilité pour leurs détenteurs d'accéder à ces catégories, trois années après leur sortie de formation, est-elle la même selon que le père est cadre ou non ?7 Par ailleurs, si effets différenciés il y a, peut-on affirmer que certains diplômes neutralisent, plus que d'autres, les impacts de l'origine sociale sur l'accès à ces catégories ? Il s'agit d'établir notamment si les diplômes professionnels de l'université (licence et master professionnels) "protègent ", plus que les diplômes généraux, leurs détenteurs des éventuels effets de l'origine sociale.

Le calcul des probabilités d'occuper, en 2007, un poste classé dans l'une de ces deux catégories ( $c f$. les deux dernières colonnes du tableau 7) révèle que, là encore, il existe assez logiquement un effet significatif du niveau et du type de diplôme. Les diplômés de bac +2 ont moins de chances d'accéder à ces catégories que ceux de licences générales (hors IUFM), tandis que les diplômés de licence professionnelle, les sortants d'IUFM, d'écoles de commerce ou d'ingénieurs et les docteurs connaissent les probabilités les plus fortes. Contrairement aux indicateurs précédents, ici, l'effet de l'origine sociale joue : être enfant de cadre augmente les chances d'occuper un emploi dans l'une de ces deux catégories. Si l'on se centre sur les seules probabilités d'occuper un poste de cadre, les effets de l'origine sociale sont également significatifs. Ainsi par exemple, les enfants d'ouvriers ont, " toutes choses égales par ailleurs ", moins de chances que les enfants de cadres d'occuper, trois ans après la fin de leurs études, un poste de cadre.

7. Ce modèle repose sur un croisement entre le plus haut diplôme et l'origine sociale regroupée en deux modalités (enfant de cadre/ enfant de non cadre). Ce croisement nous permet par exemple d'estimer la probabilité d'un jeune issu de milieu populaire et sortant de licence professionnelle d'accéder au statut de cadre. 


\section{Tableau 7}

Modèle Probit à variable dépendante (CS à 3 ans) et régression linéaire (salaire à 3 ans)

\begin{tabular}{|c|c|c|c|c|c|c|c|c|}
\hline \multirow[b]{3}{*}{ (Réf. BTS) } & \multicolumn{4}{|c|}{ En 2007, occuper un empoi de: } & \multirow{2}{*}{\multicolumn{2}{|c|}{ Salaires }} & \multirow{2}{*}{\multicolumn{2}{|c|}{ Part de: }} \\
\hline & \multicolumn{2}{|c|}{ P.I. } & \multicolumn{2}{|c|}{ Cadre } & & & & \\
\hline & Coefficient & $\begin{array}{l}\text { Test de } \\
\text { Wald }\end{array}$ & Coefficient & $\begin{array}{l}\text { Test de } \\
\text { Wald }\end{array}$ & Coefficient & $\begin{array}{l}\text { Test de } \\
\text { Wald }\end{array}$ & & \\
\hline Constante & +++ & & --- & & +++ & & P.I. & Cadres \\
\hline DUT (père non cadre) & +++ & & ++ & & +++ & & & \\
\hline DUT (père cadre) & +++ & ns & +++ & ns & ++ & ns & 56 & 11 \\
\hline Licence générale (père non cadre) & +++ & & +++ & & --- & & & \\
\hline Licence générale (père cadre) & +++ & 115 & +++ & 5 & --- & ns & 01 & 12 \\
\hline Licence pro (père non cadre) & +++ & & +++ & & +++ & & & \\
\hline Licence pro (père cadre) & +++ & ns & +++ & ns & +++ & ns & 65 & 13 \\
\hline IUFM (père non cadre) & +++ & & +++ & & +++ & & & \\
\hline IUFM (père cadre) & ++ & ns & +++ & ns & +++ & ns & 69 & 20 \\
\hline M1 (père non cadre) & ns & & +++ & & +++ & & & \\
\hline M1 (père cadre) & ns & 115 & +++ & 5 & +++ & ns & 49 & 32 \\
\hline M2 recherche (père non cadre) & -- & & +++ & & +++ & & & \\
\hline M2 recherche (père cadre) & --- & ns & +++ & $S^{\pi}$ & +++ & $S^{\pi \pi \pi}$ & 33 & 56 \\
\hline M2 pro (père non cadre) & --- & & +++ & & +++ & F & 21 & \\
\hline M2 pro (père cadre) & --- & 3 & +++ & 3 & +++ & 5 & 31 & 01 \\
\hline Ecole d'ingénieurs (père non cadre) & --- & & +++ & & +++ & & & \\
\hline Ecole d'ingénieurs (père cadre) & --- & ns & +++ & ns & +++ & ns & 10 & 81 \\
\hline Ecole de commerce (père non cadre) & ns & $*$ & +++ & & +++ & & 7 & \\
\hline Ecole de commerce (père cadre) & --- & $s$ & +++ & 5 & +++ & ns & 21 & 63 \\
\hline Doctorat (père non cadre) & --- & & +++ & & +++ & & & \\
\hline Doctorat (père cadre) & --- & $s^{*}$ & +++ & $S^{* *}$ & +++ & $s^{* * *}$ & 25 & 69 \\
\hline
\end{tabular}

Les tests de Wald sur l'égalité des coefficients nous permettent de dire si les différences observées entre les coefficients sont significatives à l'intérieur de chaque diplôme. Quand le test est significatif à l'intérieur d'un diplôme, c'est qu'un jeune dont le père n'est pas cadre a moins de chances d'être lui-même cadre trois ans après son entrée sur le marché du travail qu'un jeune dont le père est cadre.

Source : Céreq - Génération 2004 interrrogée en 2007.

Lecture : Toutes choses égales par ailleurs, les jeunes dont le plus haut diplôme est une Licence générale ont plus de chances d'accéder au statut de profession intermédiaire que les jeunes dont le plus haut diplôme est un BTS. Pour autant, au sein de cette population, l'origine sociale n'a pas d'effet significatif sur l'accès à ce statut.

Sigles : P.I. : professions intermédiaires ; BTS : brevet de technicien supérieur ; DUT : diplôme universitaire de technologie ; IUFM : Institut universitaire de formation des maîtres; $\mathrm{M1}$ : master $1 ; \mathrm{M} 2$ : master 2 . 
Il s'avère donc que, quel que soit le diplôme qu'ils possèdent, les jeunes ont plus de chances d'accéder à cette catégorie lorsque leur père est lui-même cadre. Le sens de la corrélation est toujours le même mais avec des degrés différents.

Certains diplômes «neutralisent » t-ils davantage l'effet de l'origine sociale ? Pour le savoir, nous avons calculé des " tests de Wald " d'égalité des coefficients pour chaque diplôme deux à deux ${ }^{8}$. Ces tests montrent que cette différence est significative pour les titulaires des filières disciplinaires, que ce soit au niveau licence, maîtrise, DEA-master recherche ou doctorat mais également pour ceux issus de masters professionnels et d'écoles de commerce. En revanche, ce n'est pas le cas pour les jeunes diplômés de DUT, de licence professionnelle, d'IUFM ou d'écoles d'ingénieurs; en effet, qu'ils soient ou non fils ou filles de cadres, ils ont autant de chances d'accéder eux-mêmes à un emploi de cadre trois ans après leur entrée sur le marché du travail. Ainsi, certains diplômes neutralisent plus que d'autres les effets de l'origine sociale. C'est le cas des DUT, des licences professionnelles, des IUFM et des écoles d'ingénieurs qui permettent à leurs diplômés, quelle que soit leur origine sociale, d'occuper, dans les mêmes proportions, des professions de cadres et de percevoir des salaires comparables.

Pour autant, peut-on prétendre que ces diplômes " protègent " plus que les autres leurs détenteurs des effets de leurs origines en termes de positionnement sur le marché du travail ? Il semble difficile de répondre par l'affirmative car, à l'exception des écoles d'ingénieurs qui amènent effectivement la plupart de leurs diplômés à des postes de cadres (en grande majorité sur des postes d'ingénieurs et de cadres techniques d'entreprises) et qui peuvent donc constituer, pour des jeunes d'origine sociale plus modeste, des tremplins vers la catégorie cadre, les trois autres filières (DUT, licences professionnelles et IUFM) conduisent majoritairement leurs diplômés vers des emplois classés en professions intermédiaires. Mais, hormis les écoles d'ingénieurs, les filières qui conduisent principalement au statut de cadre telles que les masters professionnels ou recherche, les doctorats et les écoles de commerce sont également celles où l'origine sociale joue un rôle significatif à la fois dans l'accès à cette même catégorie, dans le fait d' "échapper " à celle des professions intermédiaires et, dans certains cas (comme celui des masters et du doctorat), de percevoir des salaires supérieurs aux autres.

\section{Conclusion}

Cette étude montre qu'aux inégalités d'accès aux différentes filières s'ajoutent des inégalités sur le marché du travail. À diplôme égal, si l'origine sociale joue peu, voire pas, sur les modalités d'accès à l'emploi, elle semble en revanche avoir une nette influence sur la

8. Les tests de Wald sur l'égalité des coefficients nous permettent de dire si les différences observées entre les coefficients sont significatives à l'intérieur de chaque diplôme. Quand le test est significatif à l'intérieur d'un diplôme, c'est qu'un jeune dont le père n'est pas cadre a moins de chances d'être lui-même cadre trois ans après son entrée sur le marché du travail qu'un jeune dont le père est cadre. 
propension à occuper, en début de carrière, les emplois les plus valorisés. Par ailleurs, la possession de certains diplômes professionnels jusqu'au niveau licence, dès lors qu'ils y ont accès, permet certes aux jeunes dont les parents ne sont pas cadres d'accéder aux mêmes emplois que les enfants d'origine sociale plus élevée ; il n'en demeure pas moins que ces emplois sont très rarement des emplois de cadres. Ainsi, le développement des licences professionnelles, qui a permis à des enfants d'origines plus modestes qu'auparavant d'atteindre le niveau licence, ne doit pas forcément être assimilé à une « démocratisation " de l'enseignement supérieur, à l'image d'un sésame ouvrant largement à la majorité les portes vers les positions sociales les plus prestigieuses. Ce développement participe d'un allongement généralisé des scolarités, d'une augmentation du nombre de diplômés du supérieur, sans réserver à leurs détenteurs les destinées professionnelles les plus prisées. Ainsi, confondre augmentation du nombre de diplômés à ces niveaux de formation et démocratisation du supérieur serait occulter " le quasi-monopole des groupes sociaux privilégiés sur le système scolaire et sur l'accès aux filières les plus rentables socialement et professionnellement " (Garcia et Poupeau, 2003).

\section{Bibliographie}

Affichard J. (1981). "Quels emplois après l'école? La valeur des titres scolaires depuis 1973 ", Economies et Statistiques, 134.

BEAUd S. (2003), Les enfants de la démocratisation scolaire. $80 \%$ au Bac et après ?, La Découverte.

Beaud S., Convert B. (2010), " 30 \% de boursiers en grande école... et après ? ", Actes de la recherche en sciences sociales, $\mathrm{n}^{\circ} 183$, pp. 5-13.

Boumadhi R., Lemistre P. (2006), «Accès à l'emploi qualifié en début de vie active : les déterminants", Les notes du Lihre, $\mathrm{n}^{\circ} 428$.

Bourdieu P., Passeron J.-C., (1964), Les Héritiers, Les étudiants et la culture, Minuit.

Bourdieu P., Passeron J.-C. (1970), La reproduction, Editions de Minuit.

Boudon R. (1973), L'inégalité des chances. La mobilité sociale dans les sociétés industrielles, Armand Colin.

Caille J.-P. (2005), "Le vécu des phases d'orientation en fin de troisième et de seconde ", La revue Education et formations, ${ }^{\circ} 72$, MEN-DEPP.

Calmand J., Epiphane D., Hallier P. (2009), De l'enseignement supérieur à l'emploi : voies rapides et chemins de traverse, Céreq, Nef, $\mathrm{n}^{\circ} 43$.

Calmand J., Giret J.-F. (2010), L'insertion des docteurs, Céreq, Net.doc, ${ }^{\circ} 64$.

Couppié T., Epiphane D. (2008), " Les hommes et les femmes sont-ils insérés à la même enseigne? ", in Paul J.-J et Rose J. (dir), Les relations formation-emploi en 55 questions, Edition Dunod, pp. 215-220. 
Duru-Bellat M., Kieffer A. (2008), “ Du Baccalauréat à l'enseignement supérieur en France : déplacement et recomposition des inégalités ", Population, 2008/1 Vol. 63, pp. 123-157.

Epiphane D. (2002), "La féminisation de la catégorie cadres au miroir de l'insertion des jeunes ", in Arliaud M. et Eckert H. (dir.) Quand les jeunes entrent dans l'emploi..., Editions la Dispute, pp. 151-170.

Euriat M., ThÉlot C. (1995), « Le recrutement social de l'élite scolaire en France. Evolution des inégalités de 1950 à 1990 ", Revue française de sociologie, XXXVI, n³, pp. 403-438.

Felouzis G. (2000), «Repenser les inégalités à l'université », Sociétés Contemporaines, $\mathrm{n}^{\circ} 38$, pp. 67-98.

Garcia S., Poupeau F. (2003), "La mesure de la « démocratisation » scolaire. Notes sur les usages sociologiques des indicateurs statistiques ", Actes de la recherche en sciences sociales, $4{ }^{\circ} 149$, pp. 74-87.

Godet M. (1993), "La maladie du diplôme. Propositions pour une nouvelle politique », Futuribles, 173, pp. 23-46.

Goux D., Maurin E. (1997), « Démocratisation de l'école et persistance des inégalités », Economie et statistiques, $\mathrm{n}^{\circ} 306$, pp. 27-39.

Guégnard C., Calmand J., Giret J.-F., Paul J.-J. (2008), "Recognition of Higher Education Graduates' Competences on European Labour Markets”, Céreq, Training and Employment, 83.

LÉGer F., (2006), "Origine sociale, offre de formation et niveau atteint dans le secondaire ", Les notes d'information, MEN-DEPP, n06.15.

Lopez A., Joseph O., Ryk F. (2008), «Génération 2004, des jeunes pénalisés par la conjoncture ", Céreq, Bref, n ${ }^{\circ} 248$.

Merle P., (2000), « Le concept de démocratisation de l'institution scolaire : une typologie et sa mise à l'épreuve ", Population, n 55, pp. 15-50.

Merle P. (2002), La démocratisation de l'enseignement, La Découverte, Collection "Repères ".

Nakhili N. (2010), "Orientation après le bac : quand le lycée fait la différence ", Céreq, Bref, $\mathrm{n}^{\circ} 271$.

Piketty T., (2006), "Education : les promesses de la discrimination positive », Le Monde, 21 février 2006.

Prost A. (1986), L'enseignement s'est-il démocratisé ?, PUF. 